\title{
A pandemia nas fronteiras da reforma sanitária: os desafios da rede hospitalar
}

| ${ }^{1}$ Thais de Andrade Vidaurre Franco |

${ }^{1}$ Instituto de Medicina Social, Universidade do Estado do Rio de Janeiro. Rio de Janeiro-RJ, Brasil (thais.a.v.franco@gmail.com). ORCID: 0000-0001-8755-4337

Recebido em: 09/06/2020

Aprovado em: 11/06/2020

Revisado em: 20/06/2020

DOI: http://dx.doi.org/10.1590/S0103-73312020300307

A dimensão e a intensidade dos impactos de uma epidemia em determinado país resultam da combinação entre as características do agente etiológico - como modo de transmissão, patogenicidade e virulência - com a capacidade de organização e resposta de cada sociedade em diferentes momentos históricos. No caso do novo coronavírus, a suscetibilidade mundial a sua propagação expôs distintas capacidades de resposta. Essas variam em função das disputas políticas nacionais, da inserção na economia internacional, dos níveis de desigualdade social, bem como do desenvolvimento de suas instituiçóes - em especial, dos sistemas de saúde.

No Brasil, o cataclisma ético-político que atravessamos vem se revelando como o maior entrave para uma organizaçáo mínima do enfrentamento ao avanço e às consequências da pandemia. Outro agravante é que o encontro entre a pandemia e nossa estrutura de marcadas desigualdades amplia o drama das populaçóes mais vulneráveis, atingindo de forma particularmente aguda os mais pobres, as mulheres, a população negra, os trabalhadores informais e os residentes dos municípios mais pobres (BATISTA et al., 2020).

No que diz respeito aos sistemas públicos de saúde, todos seus componentes são fundamentais para o combate ao avanço da epidemia. Destacadamente, 
os serviços da Atenção Primária desempenham papel decisivo por meio da identificação e monitoramento dos casos leves, das açoóes de educação em saúde e do acompanhamento das condiçóes e agravos, que seguem necessitando de cuidados como ações de pré-natal, acompanhamento de hipertensos e diabéticos. Em todos esses casos, a ausência ou a interrupção dos cuidados pode ampliar sensivelmente a pressão sobre os serviços de internação, mostrando-se não apenas essenciais, como, talvez, o maior trunfo do Sistema Único de Saúde (SUS).

A estrutura e a capacidade de coordenação da rede hospitalar também têm se mostrado essenciais nas diferentes experiências internacionais. Relatório sobre o perfil de morbidade por Covid-19 na China estima que cerca de $14 \%$ da população acometida irá precisar de cuidados hospitalares e, 5\% de cuidados em unidades de terapia intensiva (WU; McGOOGAN, 2020). Estima-se, ainda, que o tempo médio de permanência dos pacientes que demandam leitos de UTI pode variar entre 14 e 21 dias.

Considerando esses apontamentos preliminares sobre a articulação da assistência nos sistemas de saúde, examinamos neste texto a baixa capacidade de resposta do nível de atenção hospitalar no Brasil aos efeitos da pandemia de Covid-19, situando, especialmente, a realidade do Estado do Rio de Janeiro. Para tanto, levantamos três pontos: (i) a ausência histórica de uma política de reforma da rede hospitalar e de investimentos baseada na lógica regional; (ii) a multiplicação de modelos de gestão privados, com a predominância da gerência de unidades por meio de organizações sociais (OS); e (iii) a fragmentação na gestão do sistema e, consequentemente, do cuidado.

Esses três pontos têm como eixo de organização o processo de desvalorização e esvaziamento do papel do Estado na gestáo hospitalar, fortalecendo o argumento da modernização gerencial sob predomínio da iniciativa privada. O somatório desses aspectos é ao mesmo tempo consequência e condição para a ausência da gestão baseada em uma autoridade pública sanitária que tenha o controle das diretrizes políticas e administrativas do sistema de saúde. Em oposição a esse quadro de crescente expansão de relaçôes patrimonialistas na organização da rede hospitalar, sugerimos, por fim, apontamentos gerais para um modelo alternativo baseado em uma lógica pública que garanta acesso universal e equânime em tempo oportuno para a população. 


\section{A assistência hospitalar em sistemas nacionais de saúde}

Durante as diferentes reformas dos sistemas nacionais de saúde, importantes críticas ao papel da assistência hospitalar foram elaboradas. Isso ocorreu tanto no processo de universalização do acesso e consolidação desses sistemas, na conjuntura de ampliação do Welfare State, quanto nas décadas de 1980 e 1990, no contexto das reformas neoliberais, críticas ao papel e protagonismo do Estado (ALMEIDA, 2012).

As diversas experiências internacionais de estruturação de sistemas públicos e universais, baseados na Atenção Primária à Saúde (APS), tais como Inglaterra, Espanha, Itália e Canadá, seguiram com diferentes composiçôes o modelo de redes regionalizadas. No que diz respeito à assistência hospitalar, identificou-se, nos sistemas de saúde desses países, um conjunto de tendências que articularam a redução do número total de leitos e o aumento do porte médio das unidades e densidade tecnológica. Conjugadamente, observou-se, ainda, uma queda significativa do tempo médio de permanência para os casos agudos e aumento da rotatividade de leitos (BRAGA NETO et al., 2012).

Outro aspecto relatado nos estudos trata da crescente coordenação do cuidado e integração dessas unidades às redes de serviços de saúde. Os hospitais passaram a atender os casos mais graves que exigem cuidados intensivos, assumindo a função de retaguarda e de apoio técnico à rede assistencial, além de atuar na formação de especialistas e no desenvolvimento de pesquisas (McKEE; HEALY, 2000).

No desenvolvimento do sistema de saúde brasileiro, também houve uma redução do número total de leitos, embora essa não tenha sido acompanhada pelo aumento do porte médio das unidades e do incremento tecnológico dos hospitais, como observado em outros países (BRAGA NETO et al., 2012). O que se observa é um processo de redução histórica do número médio de leitos por estabelecimento. Estudo realizado por Ugá e López (2007) verificou que, em 2005, os estabelecimentos com até 50 leitos representavam $62 \%$ da rede hospitalar brasileira e eram localizados, majoritariamente, em municípios de pequeno porte no interior do país.

Historicamente, no contexto brasileiro, os movimentos de defesa e construção do SUS se voltaram para a crítica, justa, ao modelo centralizado do INAMPS. O sistema de saúde proposto na Constituição de 1988 exigia mudanças substanciais na organização da rede de atenção à saúde. Nos anos que se seguiram, a adoção da Saúde da Família como estratégia prioritária na política nacional de saúde modificou 
o modelo de atenção brasileiro, significando um enorme avanço na garantia do direto à saúde (PINTO; GIOVANELLA, 2018). Com relação à atenção hospitalar, entretanto, diversos estudos apontam a permanência de desigualdades no acesso, indicando que mesmo após a reforma sanitária, se mantiveram a inadequação crônica do quantitativo, distribuição e perfil de leitos disponíveis, bem como a fragmentação entre esses serviços e a rede assistencial (COELHO, 2013).

Como destaca Machado (2007), apesar de os gastos com assistência de média e alta complexidade se manterem com a maior parcela do orçamento federal, em todo período de construção do SUS inexistiram políticas nacionais abrangentes direcionadas para a melhoria do acesso a esses níveis - à exceção de serviços como a terapia renal substitutiva/transplantes e oncologia. No conjunto, prevaleceu o comportamento histórico da alocação de recursos federais baseada na capacidade instalada (ALMEIDA, 2012). Segundo Coelho (2013), além da alocação insuficiente de recursos na rede hospitalar pública brasileira, outro fator que vem contribuindo para a permanência de desigualdades e distorçôes no acesso é a regionalização incompleta e tardia da saúde no país.

Como se sabe, a rede de hospitais no Brasil é composta por unidades públicas e privadas não lucrativas e lucrativas, conveniadas e náo conveniadas ao SUS. Considerando os hospitais do setor privado, é marcante a dominância de um mercado pouco regulado, com forte "tendência a concentrar-se em espaços com melhores possibilidades de sustentabilidade e retorno" (BRAGA NETO et al., 2012, p. 597). Nos últimos anos, os seguros de saúde, por seu papel de comprador de serviços, têm desempenhado papel relevante no condicionamento das tendências dos hospitais privados - lucrativos ou não (COELHO, 2013). A presença expressiva do seguimento de hospitais lucrativos no país é outra nota dissonante do que se observa nos países que dispóem de um sistema universal. No Estado do Rio de Janeiro (ERJ), por exemplo, em dezembro de 2019, dos 576 hospitais, 258 (44,8\%) eram privados com fins lucrativos.

Uma Política Nacional de Atenção Hospitalar foi publicada apenas em 2013, 25 anos após a criaçáo do SUS. No documento estabeleceu-se o papel dos entes federados no que se refere a organização e financiamento da rede hospitalar, sugerindo mudanças da remuneração por produção para a contratualização de metas qualiquantitativas de assistência, gestão e ensino/pesquisa - independentemente de sua natureza jurídica, esfera administrativa e de gestão (BRASIL, 2013). Essa política 
estabeleceu que a atuaçấo das unidades hospitalares deveria ter como referência uma população e um território definido de atuação, mas pouco ou quase nada se avançou nesse sentido desde sua publicação. Como lógica, mantiveram-se os limites históricos do processo de nossa regionalização incompleta e a manutenção de uma rede hospitalar concentrada nos grandes centros urbanos e regióes com maior renda.

Em um balanço sobre os 30 anos de construção do SUS, Gastão Wagner afirmou que a gestão de hospitais próprios e conveniados se coloca como um dos grandes desafios. Segundo o sanitarista, trata-se de uma insuficiência da capacidade instalada e de falhas na integraçáo dos serviços, que deveriam funcionar como "referência territorial e apoio para a Atenção Primária e Urgência”. Em síntese, afirma o autor, “é fundamental estender a reforma sanitária aos hospitais” (CAMPOS, 2018, p. 1.713).

No contexto atual, essa afirmação nos remete, incontrolavelmente, aos dramas decorrentes de uma autoridade sanitária débil, que encontra fortes limites para impor uma gestão coordenada dos serviços hospitalares frente ao avanço da epidemia.

\section{A assistência hospitalar no estado do Rio de Janeiro}

Como estamos apontando, a pandemia do novo coronavírus encontrou no Brasil uma assistência hospitalar segmentada, fragmentada e desigualmente distribuída no território, além de contar com uma precária coordenação e gerenciamento público. Quando consideramos o estado do Rio de Janeiro (ERJ), o cenário não é muito diferente. Por questōes histórias e econômicas, o ERJ e, principalmente sua capital, concentram um número significativo de estabelecimentos e recursos de saúde. Entretanto, por aqui também se encontram importantes vazios sanitários. Em uma análise sobre o padrão de oferta de recursos especializados nas nove regiōes do ERJ, no final da década passada, Kuschinir et al. (2010, p. 235) identificaram uma série de "carências, inadequaçôes e distorçôes em todos os níveis da rede, incluindo baixa oferta de leitos e pouco acesso à internação em algumas regióes e especialidades".

Atualmente, essas desigualdades de acesso aos serviços de média e alta complexidade permanecem. Considerando os leitos SUS de UTI, por exemplo, a proporção de leitos da Regiáo Metropolitana I era de 0,9 leito a cada10.000 habitantes, chegando a 2,6 leitos na regiáo Centro-Sul. Sobre as diferenças intrarregionais, o município de Duque de Caxias, com quase um milhão de habitantes possui uma proporção de 0,5 leito de UTI SUS a cada 10.000 habitantes (BRASIL, 2020). Esse 
quadro compromete decisivamente os esforços das secretarias estadual e municipais de saúde frente à atual crise sanitária.

Antes mesmo da chegada do vírus em terras brasileiras, a fragmentação dos serviços já impunha dificuldades para o levantamento oportuno de informaçôes sobre o número de leitos com isolamento respiratório e as possibilidades de adequação e expansão das unidades existentes. $\mathrm{Na}$ ausência de um censo hospitalar unificado e da defasagem dos sistemas de informação oficiais, o diagnóstico da capacidade ociosa e o processo de tomada de decisão sobre qual a melhor forma de organização desses serviços ficaram comprometidos.

É digno de nota que a regulação do acesso aos serviços de saúde alcançou algum avanço nos últimos anos, precisamente no que diz respeito à informatização e integração dos sistemas de informação de vagas e leitos. Não obstante essa ponderação, mantém-se a dificuldade do estabelecimento e execução da autoridade sanitária que cabe a esse serviço. Muitas vezes, o estado desempenha o papel de solicitante de vagas e, a bem verdade, até mesmo hospitais e institutos federais e universitários resistem a disponibilizarem os recursos à regulação pública.

Sem a pretensão de esgotar nesses breves apontamentos os inúmeros desafios da assistência pública hospitalar fluminense e os muitos aspectos históricos e estruturais que os configuram, cabe registrar um segundo ponto que considero parte constitutiva desses desafios, qual seja: a expansão da atuação das OS na gestão dos serviços de saúde públicos do Estado.

Nos últimos anos, a promessa de que uma mudança no modelo administrativo e organizacional executada por meio do estabelecimento de contratos de gestão com OS, possibilitaria a superação dos obstáculos estruturais e funcionais da gestão pública direta, firmou-se como dogma de uma modernidade inexorável. Como argumento, essa forma de privatização da gestão pública, tornou-se reconhecida por, supostamente, garantir uma maior flexibilidade nos vínculos de contratação trabalhista, maior agilidade na compra de insumos e na contratação de serviços (SORANZ et al., 2016). A expressiva presença dessas organizaçóes tanto na rede de serviços primários quanto na administração hospitalar exige, sem dúvida, novas investigaçôes para rastrear como essa ideologia política se consagrou como princípio dominante na gestão pública da saúde no estado e no município do Rio de Janeiro.

Segundo o site da Secretaria Estadual de Saúde, no início de junho de 2020, 14 OS eram responsáveis pela gestão de aproximadamente 30 UPAS e 15 hospitais 
estaduais. Essa alternativa, que alcançou um terreno fértil no ERJ, prevê que o estabelecimento de metas e indicadores seria suficiente para garantir um melhor desempenho da rede hospitalar. Nessa chave, não existe uma responsabilização sobre a saúde de determinada população de referência, tão pouco instrumentos que garantam uma continuidade do cuidado, ou uma governança do sistema de forma integrada. Considerando a fragmentação presente na rede de hospitais públicos do estado, a debilidade desses princípios não apenas é insuficiente para a superação dos obstáculos encontrados pela administração direta, como cria desafios adicionais.

Considerando o atual contexto de pandemia, a flexibilização dos vínculos trabalhistas, que favorece a alta rotatividade de profissionais, dificulta a garantia de continuidade de pontos importantes como a Educação Permanente, a adoção e aprimoramento de protocolos clínicos e mudanças nos processos de trabalho. Desde a reforma trabalhista de 2017, a contratação de trabalhadores como pessoas jurídicas ampliou exponencialmente a precarização e instabilidade dos vínculos, comprometendo a expertise necessária às carreiras públicas.

Um capítulo à parte, como regularmente veiculado nas grandes empresas de mídia, é a baixa capacidade de fiscalização e acompanhamento dos contratos de gestão e a ausência de transparência, que não só contribuem para possíveis desvios e maus usos da coisa pública, como inviabilizam o controle social previsto na Lei Orgânica da Saúde.

\section{Consideraçóes finais}

Neste breve comentário, buscamos argumentar que a incapacidade da rede hospitalar do ERJ de responder aos desafios impostos pela pandemia decorre, precisamente, da ausência de uma coordenação única, com base regional, capaz de planejar e exercer uma autoridade pública sanitária na organização dos recursos hospitalares. Nesse sentido, antes que identificar a ausência de instalaçóes públicas adequadas, nossa atenção se voltou para uma crítica da desconstrução sistemática do Estado.

A situação agônica a que nos referimos se localiza, sem dúvida, na fragmentação não só da rede pública, mas da segmentação do sistema de saúde nacional, que já se mostra um obstáculo para a necessária construção de uma fila única de leitos no contexto do enfrentamento da pandemia.

O cenário que identificamos se contrapóe diretamente à narrativa que localiza o "caos da rede hospitalar" no modelo de administração direta ou de uma incapacidade 
intrínseca do Estado. Como procuramos formular, os grandes desafios decorrem da ausência de uma política nacional de reestruturação da rede hospitalar no Brasil nos moldes da rede regionalizada (ou seja, a organização da rede hospitalar, também com referências territoriais - com escalas diferentes dependendo do serviço) e de uma autoridade sanitária regional. Não contribui para a extensão da reforma sanitária para a rede hospitalar o sistemático desfinanciamento a que vem sendo submetido o SUS.

Como se trata de um problema que tem certa maturação e temporalidade, no contexto dessa pandemia-dramas já conhecidos ganharam ainda mais visibilidade, e o que observamos é que a insistência nos modelos gerenciais permite a magnificação de escândalos de toda sorte - sejam eles relacionados às péssimas condiçóes de trabalho nos serviços de saúde, à improbidade e à baixa capacidade de resposta às necessidades da população fluminense. A profundidades dos desafios identificados, estamos certos, exige um debate permanente daqueles que lutam pelo SUS, agora e para além da pandemia.

\section{Referências}

ALMEIDA, C. Reforma de sistemas de saúde: tendências internacionais, modelos e resultados. In: GIOVANELlA, L. et al. (Orgs.). Politicas e Sistemas de Saúde no Brasil. 2. ed. Rio de Janeiro: Fiocruz, 2012. p. 577-608.

BATISTA, A. et al. Nota Técnica 11: análise socioeconômica da taxa de letalidade da COVID-19 no Brasil. Núcleo de Operaçóes e Inteligência em Saúde (NOIS). Disponível em: https://ponte.org/wp-content/uploads/2020/05/NT11-An\%C3\%A1lise-descritiva-doscasos-de-COVID-19.pdf

BRAGA NETO, F. C. et al. Atenção Hospitalar: evoluçâo histórica e tendências. In: GIOVANELLA, L. et al. (Orgs.). Políticas e Sistemas de Saúde no Brasil. 2. ed. Rio de Janeiro: Fiocruz, 2012. p. 577-608.

BRASIL. Portaria $n^{\circ}$ 3.390, de 30 de dezembro de 2013. Institui a Política Nacional de Atenção Hospitalar no âmbito do Sistema Único de Saúde (SUS). 31 dez. 2013, Seção 1, p. 54-56.

BRASIL. Ministério da Saúde. Cadastro Nacional dos Estabelecimentos de Saúde do Brasil CNES: Estabelecimento por tipo. Brasília. Disponível em: http:/tabnet.datasus.gov.br/cgi/ deftohtm.exe?cnes/cnv/estabbr.def Acesso em: 07 jun. 2020.

CAMPOS, G. W. S. SUS: o que e como fazer? Ciência \& Saúde Coletiva, v. 23, n. 6, p. 17071714, jun. 2018. 
COELHO, I B. Os hospitais na reforma sanitária brasileira. Tese (doutorado) - Universidade Estadual de Campinas, Campinas, 2013. Disponível em: <http://www.bibliotecadigital. unicamp.br/document $/$ ?code $=000926559>$.

KUSCHINIR, R. et al. Regionalização no Estado do Rio de Janeiro: o desafio de aumentar acesso e diminuir desigualdades. In: UGÁ, M. A. D. et al. (Org.). A gestão do SUS no âmbito estadual: o caso do Rio de Janeiro. Rio de Janeiro: Editora Fiocruz, 2010. p. 212-234.

MACHADO, C. V. Direito universal, politica nacional: o papel do Ministério da Saúde na política de saúde brasileira de 1990 a 2002. Rio de Janeiro: Editora Museu da República, 2007.

McKEE, M.; HEALY, J. The role of the hospital in a changing environment. Bulletin of the World Health Organization PMID: 10916917PMCID: PMC2560784, v. 78, n. 6, p. 803-810, 2000.

PINTO, L. F.; GIOVANELLA, L. Do Programa à Estratégia Saúde da Família: expansão do acesso e redução das internaçôes por condiçôes sensíveis à atenção básica (ICSAB). Ciência \& Saúde Coletiva, v. 23, n. 6, p. 1903-1914, jun. 2018.

SORANZ, D. et al. Eixos e a Reforma dos Cuidados em Atenção Primária em Saúde (RCAPS) na cidade do Rio de Janeiro, Brasil. Ciência \& Saúde Coletiva, v. 21, n. 5, p. 1327-1338, maio 2016.

UGÁ, M. A. D; LÓPEZ, E. M. Os hospitais de pequeno porte e sua inserção no SUS. Ciência \& Saúde Coletiva, v. 12, n. 4, p. 915-928, ago. 2007.

WU, Z.; McGOOGAN, J. M. Characteristics of and Important Lessons from the Coronavirus Disease 2019 (COVID-19). JAMA, v. 323, n. 13, p. 1239-1242, abr. 2020. 